\title{
Analysis and Research on the TD-LTE Orthogonal Frequency Division Multiplexing Modulation Technique
}

\author{
Chen Wen-ting ${ }^{1, \text { a }}$ \\ ${ }^{1}$ Chongqing College Of Electronic Engineering, Chongqing 401331, China \\ a37464351@qq.com
}

Keywords: TD-LTE, Orthogonal Frequency Division Multiplexing, Modulation Technique

\begin{abstract}
Orthogonal Frequency Division Multiplexing (OFDM) technology is a multi-carrier digital modulation technology, in recent years after high-speed digital signal processing chip (DSP) appears, so the advantages of OFDM technology is embodied. DSP technology combined with OFDM, the Fourier transform/inverse transform, QAM technology, space-time block coding, coding grid technology, soft decision and channel adaptive technology matures. OFDM technology has a good anti-multipath capability, and the flexibility and other access methods and technologies, so OFDM has been listed as a candidate for 4G wireless communication system has been widespread concern.
\end{abstract}

\section{Introduction}

With the rapid development of user demand for a variety of real-time multimedia services and increase Internet technology, people communicate quality requirements are constantly improved. Mobile communication system requires a higher quality of communication and information transfer rate. The prevailing view is that the next generation mobile communication network will be based on Ipv6 packet exchange, to provide users with peak rates up 100Mbit / s, and can support a variety of users to seamlessly roam in a wireless communication network.

According to the main technical characteristics of mobile communications is wireless access and mobile users, in order to support higher transmission rates and higher user moving speed, and effectively solve the wireless channel multipath fading and additive noise and other issues, while reducing system cost people using orthogonal frequency division multiplexing (OFDM) techniques. OFDM is a parallel multi-carrier transmission system, by extending the transmission symbol period, to enhance its ability to resist echo. Compared with traditional equalizer, its greatest feature is that a simple structure, can greatly reduce the cost, and in practical application is very flexible, high-speed digital communications is a very promising technology. OFDM technology as effectively improve the spectrum efficiency, resistance to one of the new transmission technology multipath interference, widely used in mobile communications, digital TV, digital broadcasting, and other fields, has become the most promising next generation mobile communication solutions

OFDM (Orthogonal Frequency Division Multiplexing) is a special multi-carrier modulation technology, the basic principle is the high-speed data stream through the serial parallel conversion, transmission rate assigned to the relatively low number of sub-channels for transmission , the higher the number of bits as the data stream by 1010010111000110 parallel transform $1010,0101,1100,0110$ divided into lower four bits of the data stream. Because it is through a plurality of orthogonal subcarriers parallel serial data transmission, either increase the symbol width, reduce the frequency band occupied by a single symbol, frequency selective fading caused by multipath resistance, and can effectively overcome the ISI ( inter-symbol Interference, ISI), serve to reduce the system to balance the technical requirements of the role, to apply to multi-path fading channel environment and data transmission, but also the use between carriers to further improve the frequency utilization efficiency against narrow band interference and multipath fading, it is currently one of the advanced data transmission frequency multiplexing resources, is very suitable for TD-LTE wireless broadband multiple access channel in high-speed transmission. This article sends and receives link based on OFDM system by MATLAB simulation, analysis and research 
focused on the modulation and demodulation techniques OFDM system.

\section{The Development and Application of OFDM Technology}

Orthogonal Frequency Division Multiplexing (OFDM) is a special multi-carrier digital modulation technology. The concept of OFDM originated in the 20th century, the mid-1950s. Choose one of the main reasons that the OFDM system can be a good fight against the frequency selective fading or narrowband interference. In the 1960s the concept has been formed using parallel data transmission and frequency division multiplexing.

In the 1960s, OFDM technology has already been applied to a variety of high-frequency military systems, including KINEPLEX, ANDEFT and KNTHRYN like. In KNTHRYN for example, in which the variable rate data modem can use up to 34 sub-parallel low-speed phase modulations and the interval between each sub-channel is $82 \mathrm{~Hz}$. After Weinstein and Ebert the discrete Fourier transform (DFT) is applied to the parallel transmission system to achieve the modulation and demodulation of the baseband, so that the process of achieving the FDM baseband is achieved through FDM, instead of using band-pass filters, subcarrier oscillator group and coherent demodulator.

At that time, no practical applications of powerful computer chips integrated, real-time Fourier transform complexity, stability and linearity requirements of the RF transmitter and receiver oscillator power amplifier all become conditionality OFDM technology. Thereafter OFDM technology developed slowly. Until the 20th century, the mid-80s, with the use of OFDM in the European digital audio broadcasting (DAB) program, the method began to be concerned about and has been widely used. In recent years, after the high-speed digital signal processing chip (DSP) appears, so the advantages of OFDM technology are embodied. DSP technology combined with OFDM, the Fourier transform / inverse transform, QAM technology, space-time block coding, coding grid technology, soft decision, channel adaptive technology matures.

OFDM has been in the digital audio broadcasting (DAB), Digital Video Broadcasting (DVB), based IEEE802.ll standard wireless local area network (WLAN) and a wired telephone network based on non-existing twisted pair copper symmetrical high-bit-rate digital subscriber line technology (such as ADSL) has been applied. Most of which use OFDM can effectively eliminate multipath signal propagation caused by interference between the (151) for this feature.

$\mathrm{DAB}$ is developed on the basis of analog broadcasting on AM and FM, etc., that can provide comparable with CD sound quality, as well as other new data services. In 1995, by the European Telecommunications Standards Institute (ETSI) has developed DAB standard, which is the first to use OFDM standard. Then in 1997, based on DVB standard OFDM also began to put into use. In ADSL applications, OFDM is typically treated as discrete multi-tone modulation, successfully used in a wired environment, can provide up to 8Mbit / s 54Mbit / S data rate within $1 \mathrm{MHz}$ bandwidth, which is the first time for OFDM packet communications among business. And since then, ETSI, BRAN and MMAC also have adopted OFDM as the standard physical layer.

\section{The Characteristics, Advantages and Disadvantages of OFDM}

In the high-speed data stream through the serial parallel conversion, so that the length of each sub-carrier data symbol duration is relatively increased, which can effectively reduce the time dispersion of the radio channel 151 brought, thus reducing the complexity of the receiver equalizer, and sometimes you cannot use the equalizer, not only eliminate the influence of 151 by employing a method for inserting the cyclic prefix. In order to prevent interference between the symbols, delayed OFDM system paths only a small part of the OFDM symbol length of time, as long as the length is greater than the added protection symbol multipath delay time, the interference between the symbols can be eliminated. Protection by the periodic preamble symbols, so not only can prevent interference between the symbols can be maintained and the signal periodicity. The broadband frequency selective fading channel can be viewed as a plurality of frequency flat fading narrowband channels. So that the broadband signal into a plurality of narrowband signal set by the orthogonal 
carrier transmission, so it can be frequency-selective fading channel broadband narrowband channels deemed a plurality of frequency flat fading.

The traditional frequency division multiplexing transmission method, the frequency band is divided into a number of disjoint frequency band transmission parallel data stream at the receiving end with a set of filters to separate the various sub-channels. The advantage of this method is simple and direct, the disadvantage is the low utilization of spectrum. And to achieve a plurality of filters, there are many difficulties. The OFDM system between the subcarriers, the sub-channels allows spectral overlap, as compared with the conventional frequency division multiplexing system and OFDM system can maximize the use of spectrum resources.

Each sub-channel of this modulator and demodulator can IDFT and DFT methods. For systems with large N, we can use a fast Fourier transform (FFT) to achieve. With the development of large scale integrated circuit technology and DSP technology, IFFT and FFT are very easy to achieve.

The existence of wireless data services are generally non-symmetry, that is, the amount of the down-link data transmission is much larger than the amount of uplink data transmission, such as the Internet business in the web browser, FTP downloads. On the other hand, the mobile terminal power is generally less than IW, in the large-cell environment transmission is slower than $10 \mathrm{kbit} / \mathrm{s}$ a $100 \mathrm{kbit} /$; s transmission power of the base station can be larger, it is possible to provide $1 \mathrm{Mbit} / \mathrm{s}$ transfer rate above. Therefore, whether from the use of user demand for data services, or from a mobile communication system needs to consider, and hope the physical layer to support non-symmetric high-speed data transmission, and OFDM system can easily be done by using a different number of sub-channels to achieve uplink and downlink link different transmission rates.

Due to the extent of the decline suffered by each sub-carrier is not necessarily the same as a part of the carrier may suffer more serious decline, usually with FEC. Coding techniques to correct the error signal when the retransmission signal on the original and on different sub-carriers. Due to the existence of frequency selective radio channel, all subcarriers are impossible while in a relatively deep fading, so you can dynamically allocate bits and dynamic sub-channel allocation method, take full advantage of high SNR sub-channels, thereby improve system performance. But also for multi-user system, the user does not apply to a sub-channel for another user, it may be relatively good performance of the sub-channel, so unless a sub-channel for all users are applicable, only the sub-channel It was closed, but the probability of this happening is very small.

OFDM systems can easily access a variety of other methods used in combination. Constituting OFDMA systems, including multi-carrier CDMA MC-CDMA, frequency hopping and OFDM OFOM-TDMA, etc., so that multiple users can simultaneously use OFDM technology for the transmission of information.

Since narrowband interference affects only a portion of sub-carriers, so the OFDM system can resist such narrowband interference in some way. In a single-carrier system, such as the GSM system, various fading channels will be willing to disruption of the entire communication, but in a multi-carrier system, because each carrier confrontation with the decline interrupted by chance is very small, so it can be error correction coding to correct these errors.

\section{The OFDM System Modulation Simulation Technology}

To simulate the process OFDM modulation system, the author set up eight test points, respectively, and then the test signal that eight points, and the manner shown comparative analysis of the changes and send the link receive chain signal. In the simulation process, omit the encoding interleaving, pilot inserting synchronization signal insertion and digital to analog conversion stub process does not affect the simulation results. In addition, the whole process simulation of MATLAB also needs to do the following:

To determine the relevant parameters: number of subcarriers 200, FFT length $\mathrm{N}$ 512, per subcarrier symbol number is 14 , the guard interval length is 128,4 bits per symbol, signal to noise ratio of 15 ;

It determines that the input data: generates random binary data, the number of data generated each time $=$ the number of subcarriers per subcarrier symbol number $\times \times$ bits per symbol. Sending 
binary data bits is generated by the random-ended input, to facilitate observation only take the first 50 bits.

Sub-carrier baseband modem: total OFDM uses BPSK, QPSK, 16QAM, 64QAM demodulation four modulation, 16QAM scheme used in this simulation, and thus each OFDM symbol has 4 bits per subcarrier, the corresponding constellation 16 points.

IFFT transform: 16QAM baseband modulation on each subcarrier mapped data to a plurality of positions of the constellation points after converted into two-phase and quadrature components of the two components during operation need to synchronize signals are formed sample discrete-time signal. Because the bandwidth of the OFDM signal is $B=N * \triangle f$, the signal must $\triangle t=1$ / $B=$ $1 /(\mathrm{N} * \triangle \mathrm{f})$ sampling interval. According to Nyquist theorem, the sampling points are equal to 2 times the sub-carrier of the highest order. Here IFFT sampling points is 512, using MATLAB simulation of its function, simple operation.

Adding CP: the in-phase and quadrature components of each symbol after IFFT operation are converted into serial data, and set the length of the tail symbols of the data added to the header, thereby forming a cyclic prefix CP.

In series with noise: receiving a link is the reverse process of sending the link. After receiving antenna receives signals from the transmitting antennas, actually string into a noise simulation using the white noise superimposed on the signal directly transmitting antenna, since white noise is added on the whole number of bits, so adding CP the signals do not matter much.

Each section receive chain simulation: receiving a link in each process is just the reverse process of sending link corresponding step.

\section{Conclusions}

OFDM is a high-speed multi-carrier modulation, via the serial high speed data signal into parallel low-speed sub-data stream, and then use a set of mutually orthogonal sub-carriers of the sub-transmission of all the sub-channels are narrowband It can be considered flat fading. Therefore, OFDM system has a strong anti-frequency selective fading, multipath characteristics, narrowband interference and high spectral efficiency. OFDM has been successfully applied to the power line communications, digital audio broadcasting (DAB), digital terrestrial TV broadcasting, wireless LAN, high-speed digital subscriber line and other fields. It is considered to be one of the key fourth-generation broadband mobile communication systems.

\section{References}

[1] Huifang Zhou: Electronic Information Technology, Vol. 6 (2014) No 53, p.25-26

[2] Hongli Zhang: Data Communication, Vol. 12 (2012) No 27, p.74-76

[3] Qin Guo: Mobile Communications, Vol. 1 (2011) No 33, p.11-14

[4] Jieming Liu: Guangxi Normal University, Vol. 3 (2012) No33, p.121-124

[4] Yin Changchuan: Electronic Information Technology, Vol. 3 (2014) No33, p.58-63 